\title{
SIMULATION OF PRODUCTION LINES - THE IMPORTANCE OF BREAKDOWN STATISTICS AND THE EFFECT OF MACHINE POSITION
}

\author{
Ilar, T. ; Powell, J. ${ }^{* *} \&$ Kaplan, A. \\ * Luleå University of Technology, SE97634 Luleå, Sweden \\ ${ }^{* *}$ Laser Expertise Ltd., Nottingham, NG7 2TR, UK \\ E-Mail: ilar@ltu.se; jpowell@laserexp.co.uk; alka@ltu.se
}

\begin{abstract}
This paper demonstrates the importance of choosing the correct values and statistical distributions for breakdown frequency and duration when simulating production line productivity. Statistical distributions with a wide range tend to reduce the productivity of the line but this trend can be disrupted by poor choice of mean values for the variables in question. Also, it is demonstrated that the productivity of a production line can be improved simply by re-arranging the order of unreliable machines in the line. If the line consists of similar or exchangeable machines, productivity can improved if the most unreliable machines are placed towards the end of the line. The paper also demonstrates the risks of reduction of the standard deviation to obtain a more deterministic model.

(Received in December 2007, accepted in July 2008. This paper was with the authors 2 months for 1 revision.)
\end{abstract}

Key Words: Simulation, Manufacturing, Breakdown, Statistic Distribution, Scrapping

\section{INTRODUCTION}

Some of the effects of machine breakdowns on productivity are modelled in commercially available simulation software. However, as earlier work by the present authors suggests [1], the treatment of machine failure is often oversimplified and this can lead to misleading simulation results. For example, simulation models do not generally link the scrapping of components to machine breakdowns. In real production situations however, the item being produced when the machine breaks down is often scrapped. This is because many engineering operations (casting, welding, bending, etc.) cannot be completed successfully once they have been interrupted. This paper demonstrates that a useful mathematical analysis of the breakdown behaviour of a machine must be supported by a carefully chosen statistical description of that behaviour in the context of the production line.

The application of computer simulation for modelling production disturbances has been discussed in several papers [2-6]. For example, the work of Ingemansson et al [7, 8] often includes references to machine breakdowns and part scrapping but the two phenomena are not directly linked. The importance of the correct interpretation of breakdown characteristics in achieving high model accuracy has also been demonstrated [9]. However, this interpretation only effects secondary performance measures (i.e. buffer size and product lead time) and not, as in our approach, the main performance measure - productivity.

The reduction of standard deviation in the statistical distribution applied in the model is method to perform validity tests of the model [10] or in general to obtain a deterministic model to increase the interpretation opportunities. 


\section{SIMULATION EXPERIMENTAL WORK}

Production simulation software packages offer a range of statistical distributions for input variables. These statistical approaches are intended to give a realistic model of the behaviour of a system.

In this investigation a production simulation tool (Enterprise Dynamics) was used to analyse the productivity of the production line shown in Fig. 1 using chosen mean values for Mean Time To Failure (MTTF), Mean Time To Repair (MTTR) and Process Time (PT) for the various stations. In order to compare the results of various statistical distributions the mean values were employed in three ways; 1 . As a fixed value, 2 . As the mean of a triangular distribution and 3 . As the mean of various Gaussian distributions.

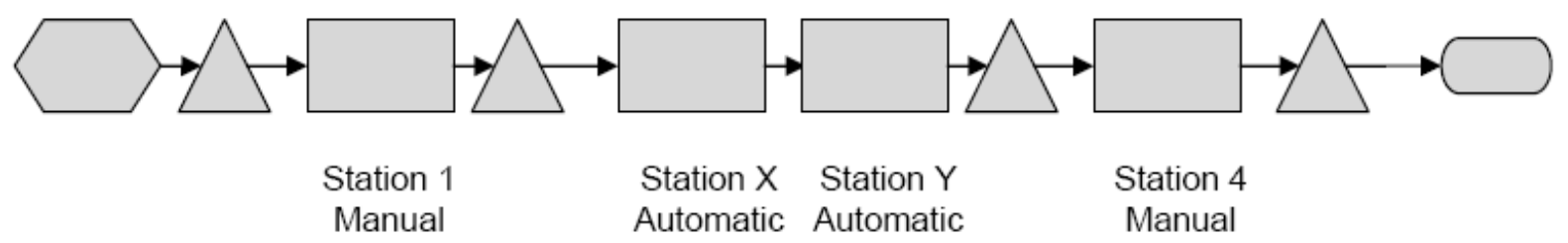

Figure 1: The production line under investigation.

The situation being considered is as follows; Stations $\mathrm{X}$ and $\mathrm{Y}$ are identical machines carrying out similar operations (eg weld 1 and weld 2). This is quite a common layout in industry and is applicable, for example, in spot welding lines for automotive body in white production or a line of machining centres performing different metal cutting operations. Both machines are somewhat unreliable but machine $\mathrm{X}$ breaks down more often than machine $\mathrm{Y}$ and also takes longer to repair on each occasion. The management of the company in question are considering replacing one or both machines with faster, more reliable machines (Q).

The simulations were carried out for 400 hours with 5 replicates. The availabilities for Stations Y and Q were set to $95 \%$ and $99 \%$ respectively, the availability of station X was initially set at $85 \%$ although other values are used for comparison later in the discussion. Table I describes the parameters used in the simulations.

Table I: Parameters employed in the comparison of different distributions (all times are in minutes).

\begin{tabular}{|c|c|c|c|}
\hline & \multicolumn{3}{|c|}{ Distributions [min] } \\
\hline Parameter & Fixed Mean Time & Triangular & Gaussian \\
\hline Arrival & Fixed; no constraints & Fixed; no constraints & Fixed; no constraints \\
\hline Process time / Station 1 and 4 & Fixed; 10.0 & $\mathrm{~T}(10,5,15)^{1)}$ & $G(10,5)^{2)}$ \\
\hline Process time / Station $\mathrm{X}$ and $\mathrm{Y}$ & Fixed; 10.0 & Fixed 10 minutes & Fixed 10 minutes \\
\hline Process time / Station Q & Fixed; 8.0 & Fixed 8 minutes & Fixed 8 minutes \\
\hline MTTF Station X (85 \% ok) & Fixed; 40.8 & $\mathrm{~T}(40.8,20.4,61.2)^{1)}$ & $\mathrm{G}(40.8,20.4)^{2)}$ \\
\hline MTTR Station X (85 \% ok) & Fixed; 7.2 & $\mathrm{~T}(7.2,3.6,10.8)^{1)}$ & $\mathrm{G}(7.2,3.6)^{2)}$ \\
\hline MTTF Station Y (95 \% ok) & Fixed; 57.0 & $\mathrm{~T}(57,28.5,85.5)^{1)}$ & $\mathrm{G}(57,28.5)^{2)}$ \\
\hline MTTR Station Y (95 \% ok) & Fixed; 3.0 & $\mathrm{~T}(3,1.5,4.5)^{1)}$ & $\mathrm{G}(3,1.5)^{2)}$ \\
\hline MTTF Station Q (99 \% ok) & Fixed; 237.6 & $\mathrm{~T}(237.6,118.8,356.4)^{1)}$ & $\mathrm{G}(237.6,118.8)^{2)}$ \\
\hline MTTR Station Q (99 \% ok) & Fixed; 2.4 & $\mathrm{~T}(2.4,1.2,3.6)^{1)}$ & $\mathrm{G}(2.4,1.2)^{2)}$ \\
\hline
\end{tabular}

Note that the PT, MTTR and MTTF distributions for the different stations are not based on real data. The values given here were chosen to demonstrate the various effects under 
discussion in this paper. Also observe that the Gaussian distribution can result in negative values when the standard deviation is high. This effect is controlled in the simulation by setting any negative result to zero (so called truncation).

Fig. 2 gives the result of the simulation for three different types of statistical distribution:

a. Fixed Mean Times (FMT) - No statistical distribution - mean values only.

b. Triangular (triang) - In this case we have triangular distributions with the same mean times as above but a minimum of half the mean and a maximum of one and a half times the mean.

c. Gaussian (Gauss) - In this case we consider Gaussian distributions with the same mean values as above and standard deviations of half the mean value.

These three types of distribution were compared against each other for all possible combinations of the machines ; two new machines (QQ), the replacement of either of the old machines with a new machine in all the arrangements possible (QY, YQ, QX or XQ) and both arrangements of the old machines if no replacement takes place. In Fig. 2 the results have been presented in order of decreasing productivity.

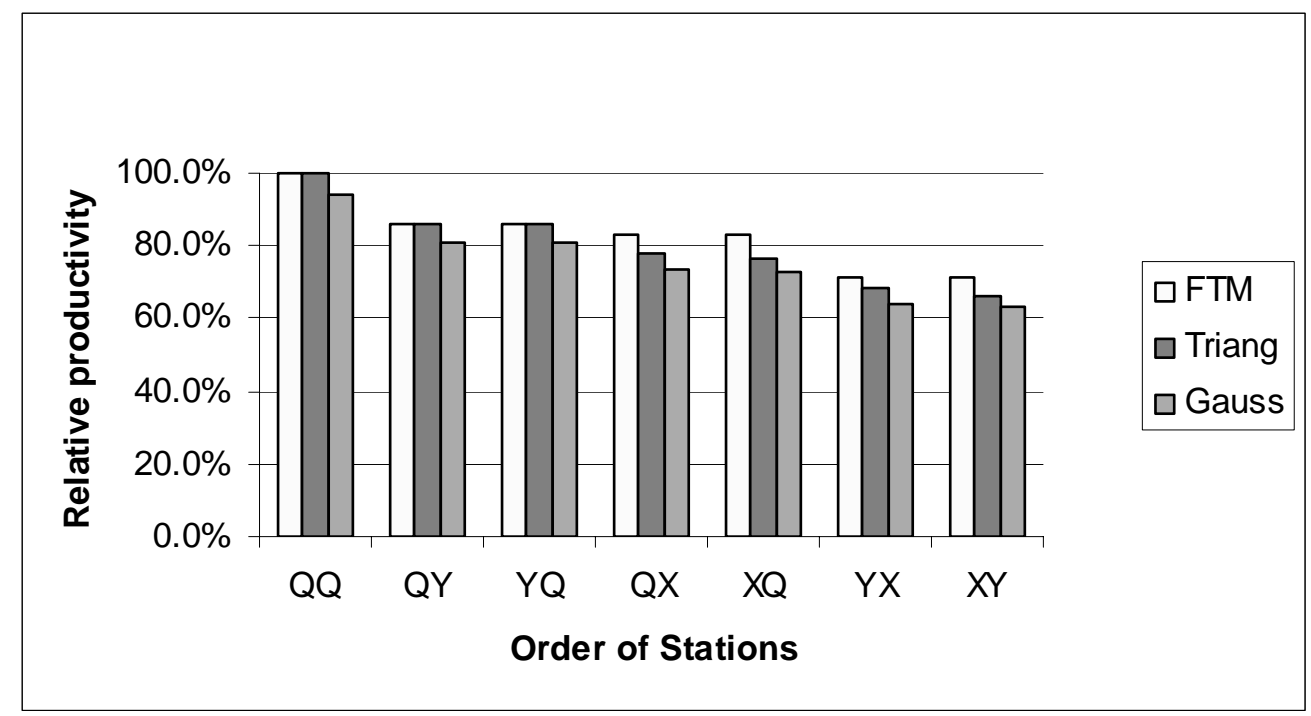

Figure 2: The relative productivity of different arrangements of the stations.

The availability for station $\mathrm{X}$ is $85 \%$, Y is $95 \%$ and Q is $99 \%$.

Fig. 2 demonstrates several points, two of which are obvious and others which need some discussion:

The obvious points;

1. Replacing both $\mathrm{X}$ and $\mathrm{Y}$ with new machines gives the highest productivity.

2. Replacing $X$ with a new machine improves productivity more than replacing $Y$.

The more interesting points;

\section{A. The order in which two similar machines appear in the line affects productivity if the machines are of different reliability;}

Close examination of Fig. 2 reveals that the order in which the stations are arranged has an effect on line productivity. This effect can be seen more clearly in Fig. 3 which shows, for example, that changing the machine order from XY to YX improves the productivity of the line by over $2.5 \%$ for the triangular distribution and by over $1.5 \%$ for the Gaussian. This point, that line productivity improves if the more reliable machines precede the unreliable ones, is confirmed in Figs. 4 and 5 which consider the same production line if the availability 
of machine $\mathrm{X}$ is reduced to $67 \%$ (MTTF; 35 mins, MTTR; 17.5 mins). In this case we can see that the productivity of the line increases by almost $10 \%$ for the triangular distribution if machine $\mathrm{X}$ follows $\mathrm{Y}$ rather than preceding it. The sensitivity of the line to machine order is reduced if more reliable machines are considered. For example, for the QY/YQ comparison, productivity changes by less than $1 \%$ for all the distributions examined in Fig. 5.

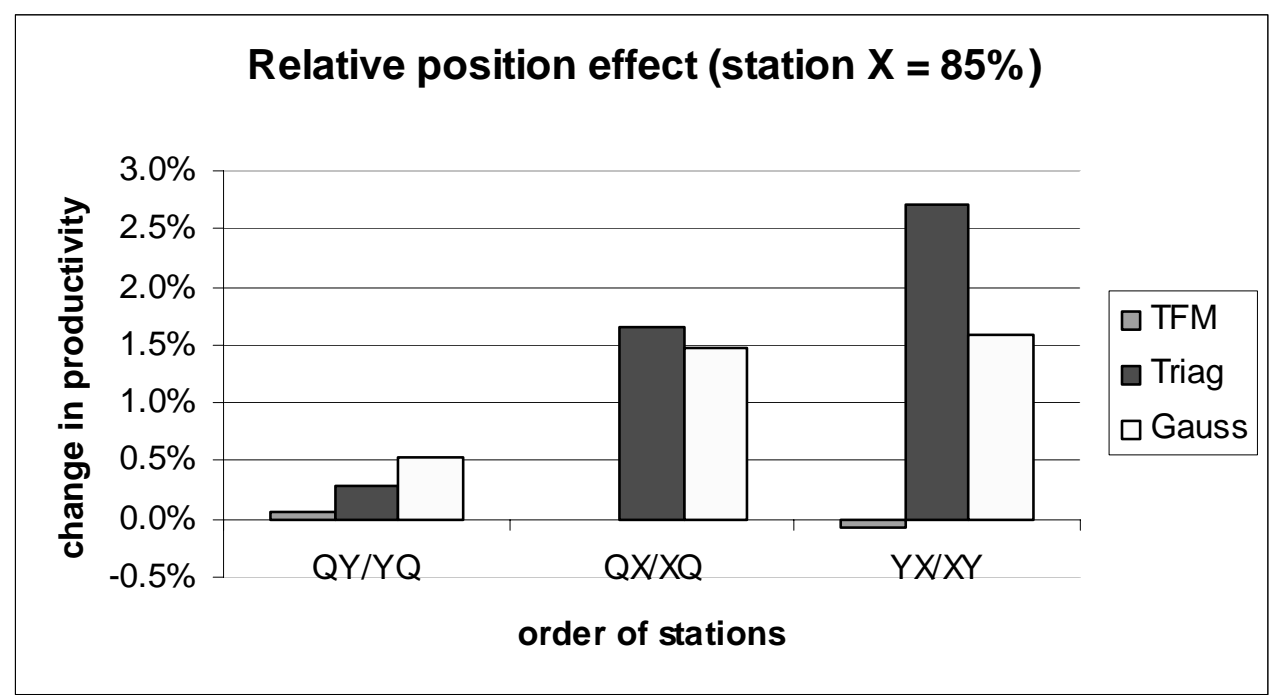

Figure 3: The changes in line productivity achievable if the less reliable machine is placed later in the line rather than earlier; eg. the $\mathrm{YX} / \mathrm{XY}$ value is calculated by dividing the line productivity if the machines are arranged ' $Y$ then $X$ ' by the productivity of the ' $\mathrm{X}$ then $\mathrm{Y}$ ' arrangement ( $\mathrm{Y}$ being the more reliable machine).

Results are given for each of the statistical distributions presented in Table I.

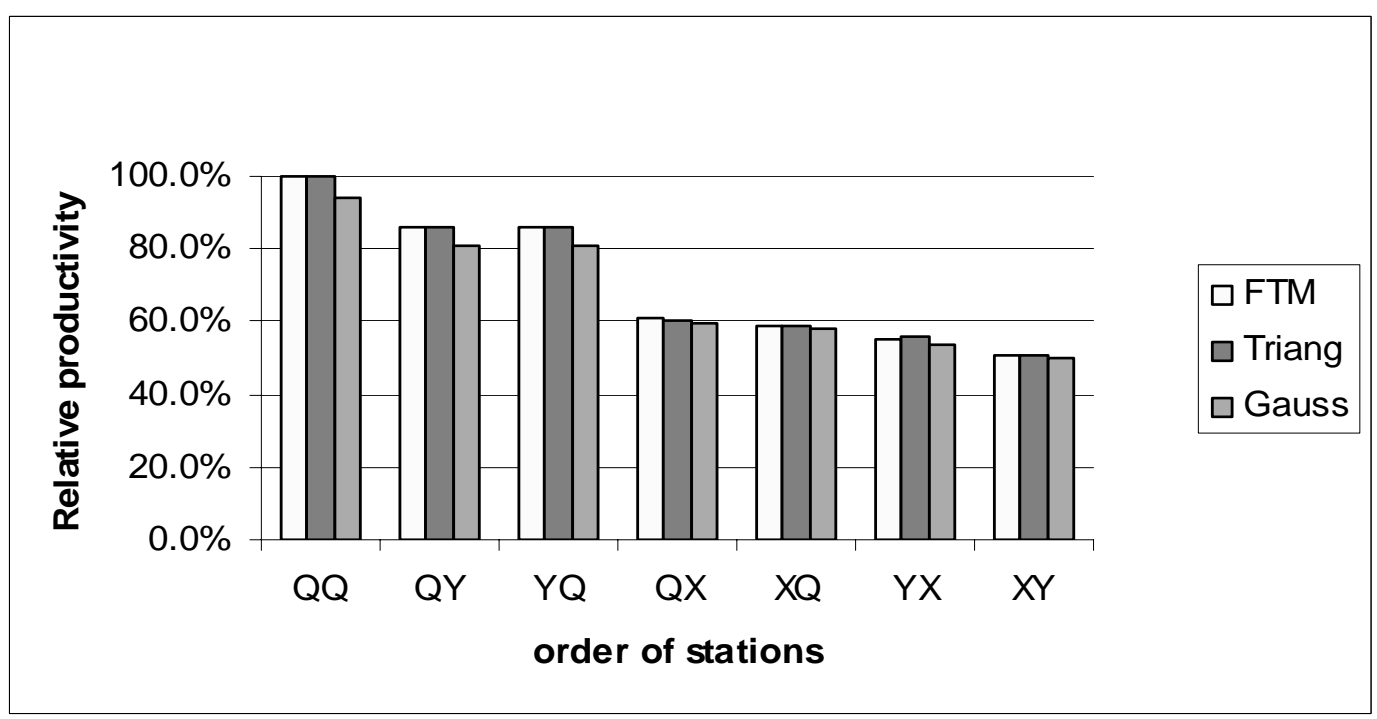

Figure 4: The relative productivity of different arrangements of the stations if the availability for station $\mathrm{X}$ is reduced to $67 \%$. 


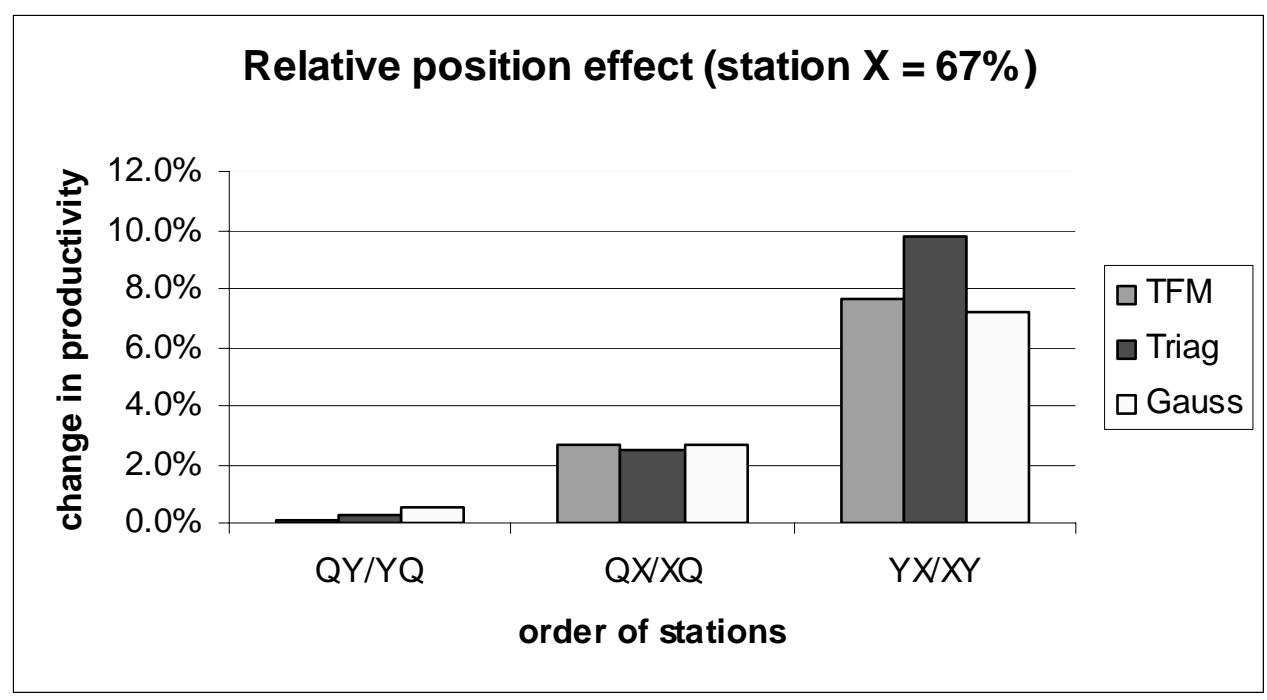

Figure 5: The changes in line productivity achievable if the less reliable machine (X machine - with an availability of $67 \%$ ) is placed later in the line rather than earlier; eg. the $\mathrm{YX} / \mathrm{XY}$ value is calculated by dividing the line productivity if the machines are arranged ' $\mathrm{Y}$ then $\mathrm{X}$ ' by the productivity of the ' $\mathrm{X}$ then $\mathrm{Y}$ ' arrangement ( $\mathrm{Y}$ being the more reliable machine).

The clearest way to explain the effect of machine order on line productivity is to describe what happens if both $\mathrm{X}$ and $\mathrm{Y}$ machines break down at the same time. We will, in the interests of clarity, use fixed mean values from table 1 for the following discussion;

XY machine order - simultaneous breakdown;

$\mathrm{X}$ takes 7.2 minutes to repair and $\mathrm{Y}$ takes 3 minutes. As $\mathrm{X}$ is before $\mathrm{Y}$ in the line, production on $\mathrm{Y}$ must wait until a part arrives from $\mathrm{X}$. From the start of the breakdown machine Y must wait 17.2 minutes before starting work - and the component arrives at station 4 (see Fig. 1) 27.2 minutes after the breakdown.

YX machine order - simultaneous breakdown;

$Y$ takes 3 minutes to repair and can begin processing a new component after this time. The repair to $\mathrm{X}$ continues whilst $\mathrm{Y}$ is operating and is finished before $\mathrm{Y}$ completes its process. In this case the second machine of the two $(\mathrm{X})$ begins work after 13 minutes and the item arrives at station four 23 minutes after the breakdown began (a saving of 4.2 minutes production time compared to the XY case).

This ability of an unreliable machine to 'starve' subsequent machines of work is the reason why the positioning of the most unreliable machines towards the end of the line improves productivity. Of course this deliberate arrangement of the machines is not always possible, but it should be considered whenever similar or exchangeable machines are part of a production line.

One more minor reason why productivity can be improved if the more unreliable machine is placed later in the line is demonstrated in Fig. 6, which graphically represents the performance of machines $\mathrm{X}$ and $\mathrm{Y}$ in both their combinations. In this case the reliability of machine X is 75 \% (MTTF 40.8 mins, MTTR 13.6 mins) and that of $\mathrm{Y}$ is $95 \%$ (MTTF 57 mins, MTTR 3 mins). Fig. 6 shows us that if machine $X$ is placed before machine $Y$, then the full effect of breakdowns on both machines limits productivity. If, on the other hand, machine $\mathrm{Y}$ precedes machine $\mathrm{X}$, then the machine $\mathrm{Y}$ breakdowns do not have much effect on productivity because they happen during the times when machine $\mathrm{X}$ is being repaired. 
Obviously the parameters have been chosen here to present an extreme case, but it is possible to have short breakdowns which do not affect production if they happen during longer breakdowns on other machines. This effect will become more noticeable for very unreliable machines where breakdown overlap will be more common.

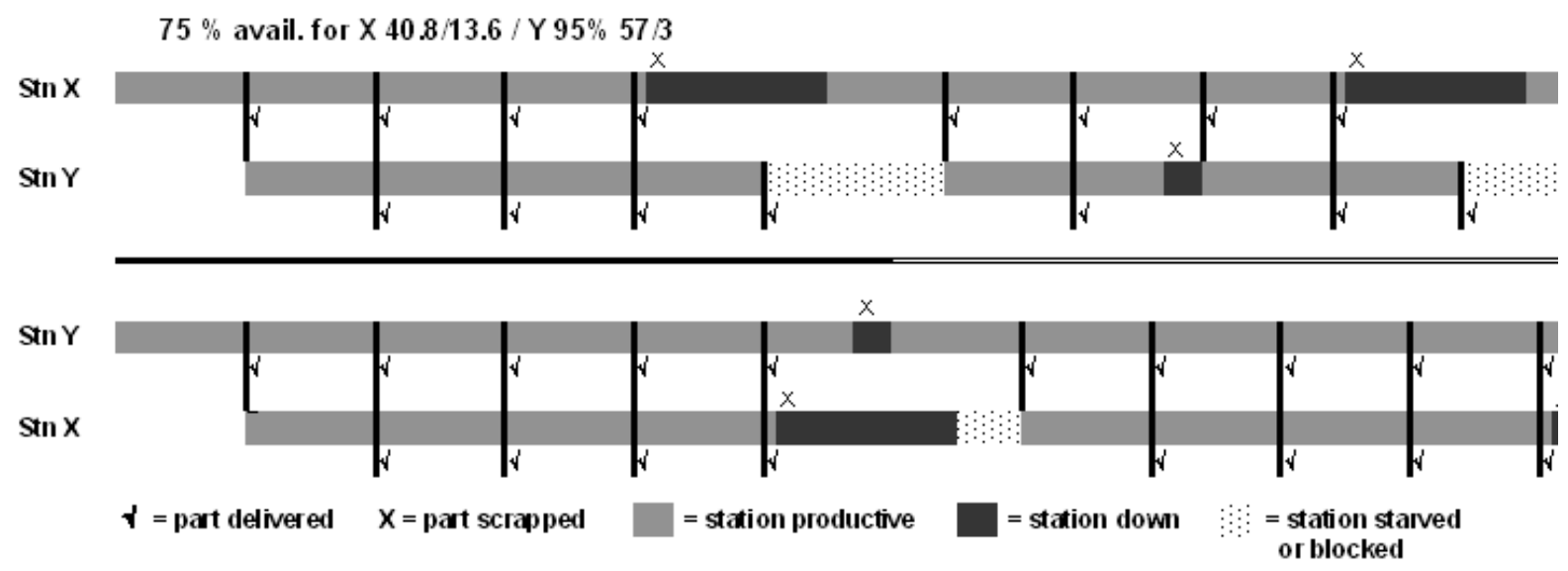

Figure 6: A comparison of XY and YX machine order demonstrating that short breakdowns on one machine can be hidden behind long breakdowns on another.

\section{B. Different statistical models using the same average values give different productivity results;}

For each machine combination shown in Fig. 2 there is a hierarchy of productivity for the different types of statistical distribution; Fixed mean times generally give the best productivity followed by the triangular, then the Gaussian distributions. As all these approaches involve the same mean values for MTTF and MTTR this hierarchy requires some explanation;

If the triangular and Gaussian distributions describe a long period of production this will include shorter periods of high and low productivity for each machine (as the frequency of breakdowns and time to repair change temporarily). For the statistical distributions involved here, there will, of course, be an equal amount of matching high and low productivity periods centred around the mean values for MTTF and MTTR. However, when the whole production line is considered, the high and low productivity periods for the individual machines do not cancel each other out. A temporary increase in breakdown rate for a particular machine is likely to reduce the production rate of the line but a temporary decrease in breakdown rate for a given machine will not always increase production. In this case for example, a period of exceptionally poor performance by machine $\mathrm{X}$ will probably result in $\mathrm{X}$ becoming the rate determining machine. However, a period of unusually good performance by machine $\mathrm{X}$ may be simultaneous with a period of poor performance by machine $Y$. If this happens machine $Y$ might become rate determining and the balancing effect of X's improved performance will be lost. The difference in productivity between the triangular and Gaussian distributions shown in Fig. 2 is due to the wider spread of MTTR and MTTF values allowed by the Gaussian distribution (see Fig. 7) - the occasional periods of very high breakdown rate or longer repairs possible for individual machines in the (broader scope) Gaussian distribution will restrict overall productivity. This point, that a wider statistical distribution of MTTR and MTTF results in lower productivity, is supported by Fig. 8, which compares productivity for a Gaussian distribution with different standard deviations. 


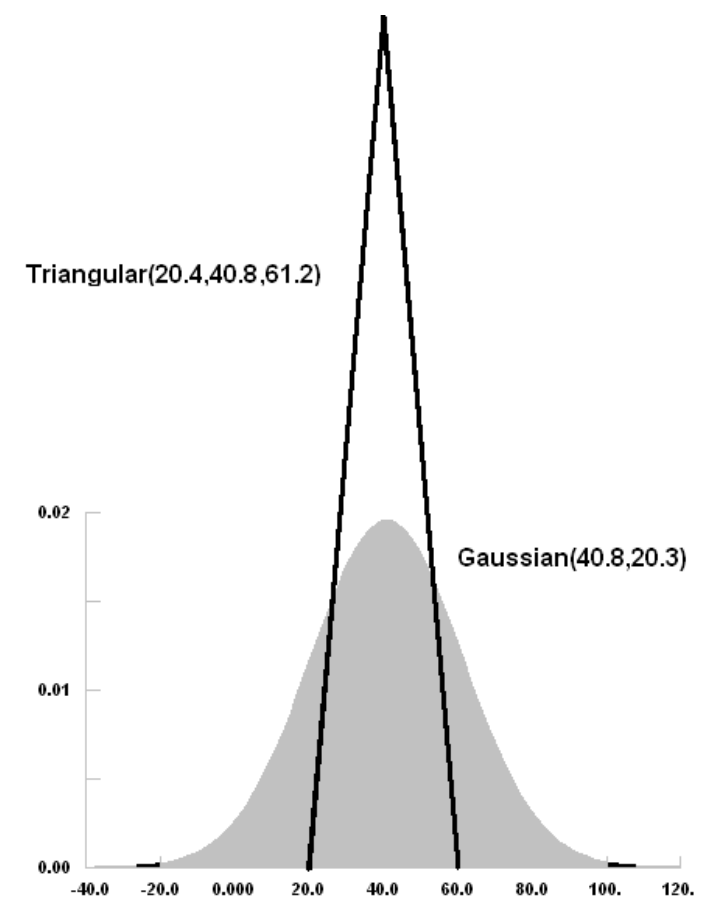

Figure 7: A comparison of the spread of values available for the triangular and Gaussian distributions being considered in Table I.

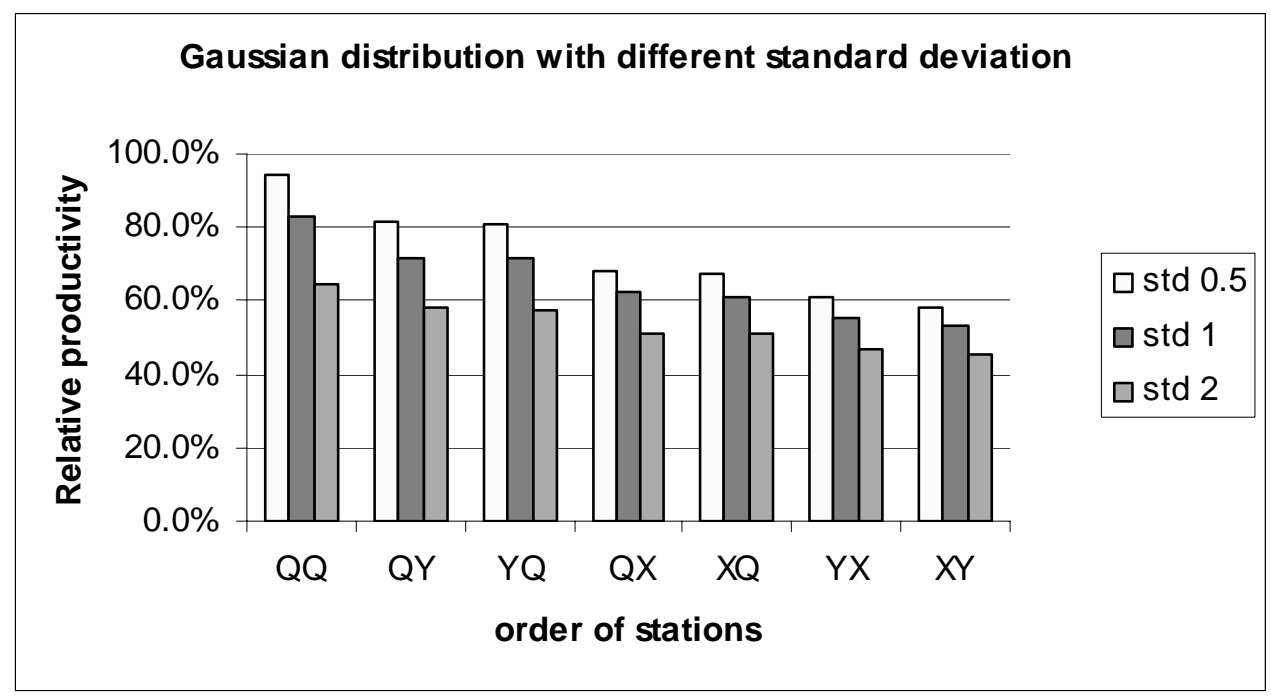

Figure 8: The productivity of the line is reduced as the spread of values for MTTR and MTTF increases (ie as the standard deviation of the Gaussian distribution increases).

\section{The importance of careful choice of mean values}

Although Fig. 8 indicates that productivity decreases as the spread of possible values of MTTF and MTTR increases (ie As the standard deviation increases), this trend can be disrupted by, for example, small changes in MTTF and MTTR;

Fig. 9 presents results of the relative performance of the production line (with machine $Y$ before X) as a function of increasing standard deviation (of MTTF and MTTR) for three different percentage availabilities for machine X. It is clear that, in general, the productivity of the line decreases with increasing standard deviation - as in Fig. 8. 


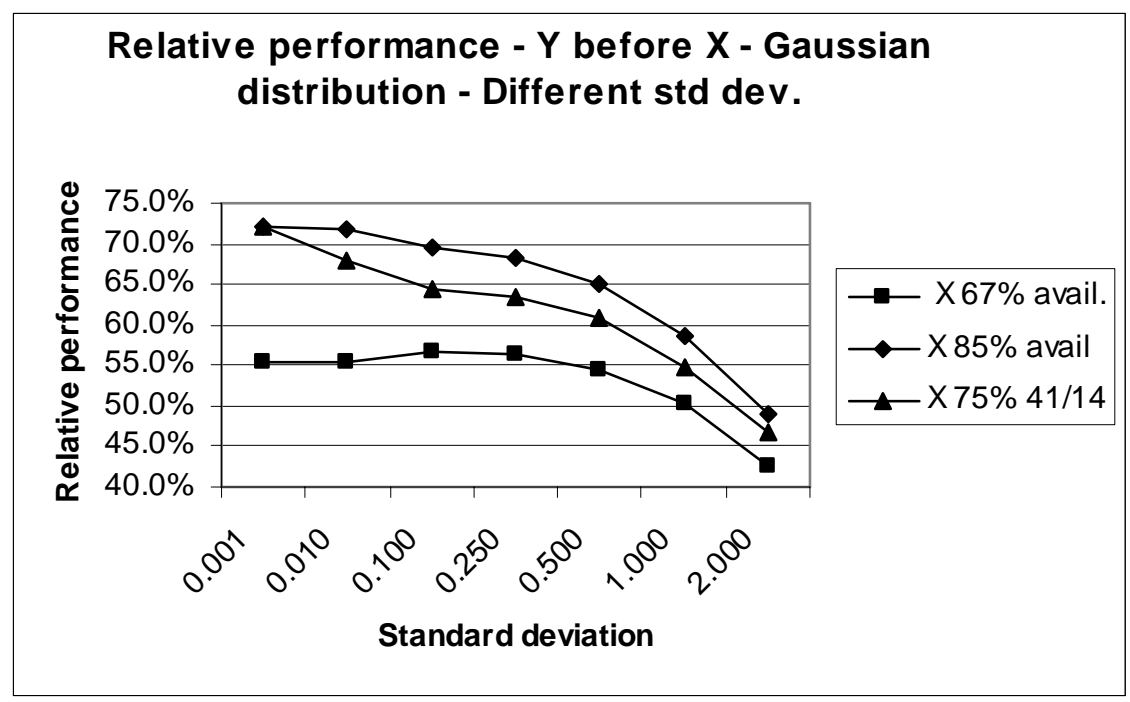

Figure 9: Although the general trend is for decreasing performance with increasing standard deviation, this trend is not always reliable - see Fig. 10.

Fig. 10 however, demonstrates that, in the $75 \%$ availability case at low standard deviation, it is possible to decrease the relative productivity of the line from $72 \%$ to $55 \%$ simply by changing the values of MTTF and MMTR from 41 and 14 minutes respectively to 39 and 13.

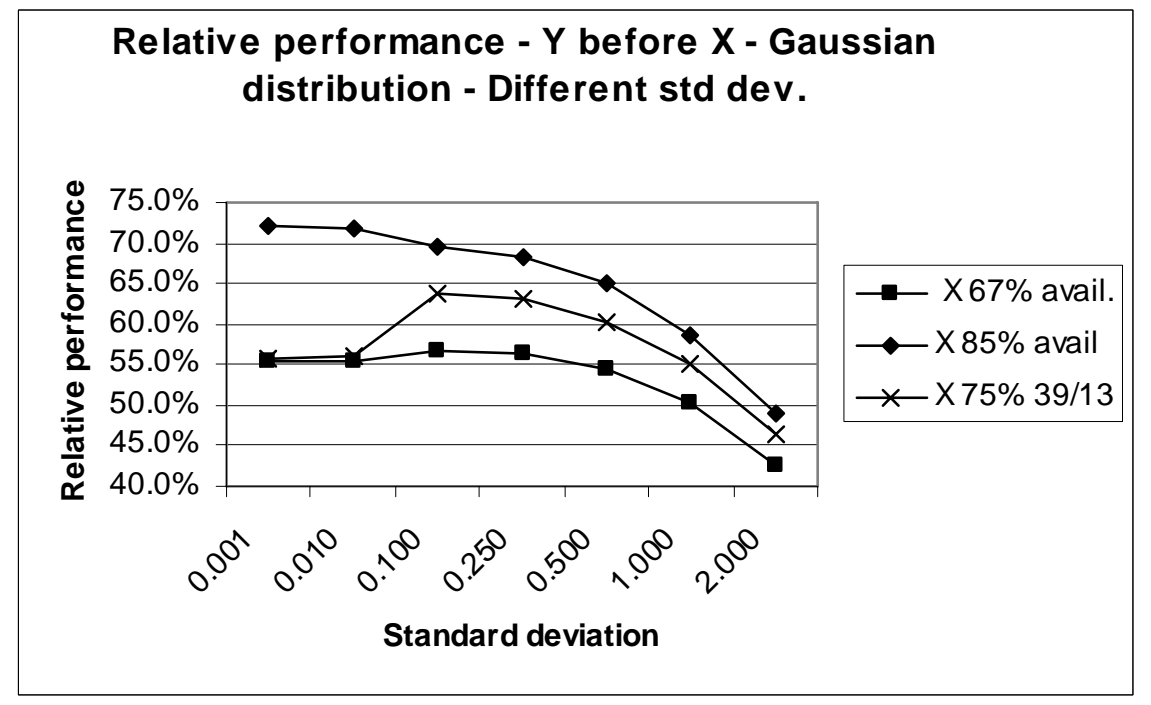

Figure 10: The general trend for decreasing productivity with increasing standard deviation can be disrupted at small sd values by small changes in the values of MTTF and MTTR. Here, in the $75 \%$ availability case, the MTTF has been changed from 41 (Fig. 9) to 39 and MTTR has decreased from 14 to 13. As a result the relative productivity has dropped from $72 \%$ (Fig. 9) to $55 \%$.

On the face of it this is an astonishing result but it is easily explained as follows;

First of all we can make the assumption that the item in the machine at the time of the breakdown will be scrapped and the time spent on processing it up to the breakdown will be wasted. Earlier work by the authors [1] has established that this is a reasonable assumption for most engineering processes (because most engineering processes cannot be interrupted). We 
have a unit time (PT) of 10 minutes. If the machine breaks down after 39 minutes of production then it will have produced three good pieces and one scrap piece - which has involved 9 minutes of wasted production time. If the machine breaks down after 41 minutes it will have produced four good pieces and one scrap piece - which has only involved only one minute of wasted production time. It is clear from this that the 41/14 MTTF/MTTR combination will be far more productive than the deceptively similar 39/13 one if the system runs on fixed mean time values.

In the case of the 39/13 minute combination, the productivity of the line is low at small values of standard deviation (see Fig. 10). This is primarily as a result of the unfortunate relationship between PT and MTTF described above. At larger values of standard deviation the 39/13 and 41/14 lines converge because the spread of results increases until the two mean values become indistinguishable i.e. at very low values of standard deviation MTTF and MTTR are effectively fixed values, and in this case, 39 minutes will always coincide with the final minute of production of a part. Similarly, 41 minutes will always be associated with the first minute of production of a part. As the standard deviation increases, the values for either 39 or 41 might be any where between, say 36 or 44 - so either mean figure might involve a failure at the beginning or end of a production operation.

\section{Unexpected results}

As a result of the interplay of the variables involved we can occasionally get unexpected results such as those shown in Fig. 11. This figure demonstrates the effect of XY/YX machine order on line productivity for different $\mathrm{X}$ machine availabilities over a range of standard deviations. As well as the conflicting trends given by the choice of mean value (discussed above) there are numerous unexpected 'kinks' in the lines on the graph - some of which are counterintuitive (particularly in the $75 \% 41 / 14$ case). It is not the intention of this paper to investigate such 'kinks' in detail - we simply want to draw the attention of workers in this field to the difficulties involved in identifying reliable trends.

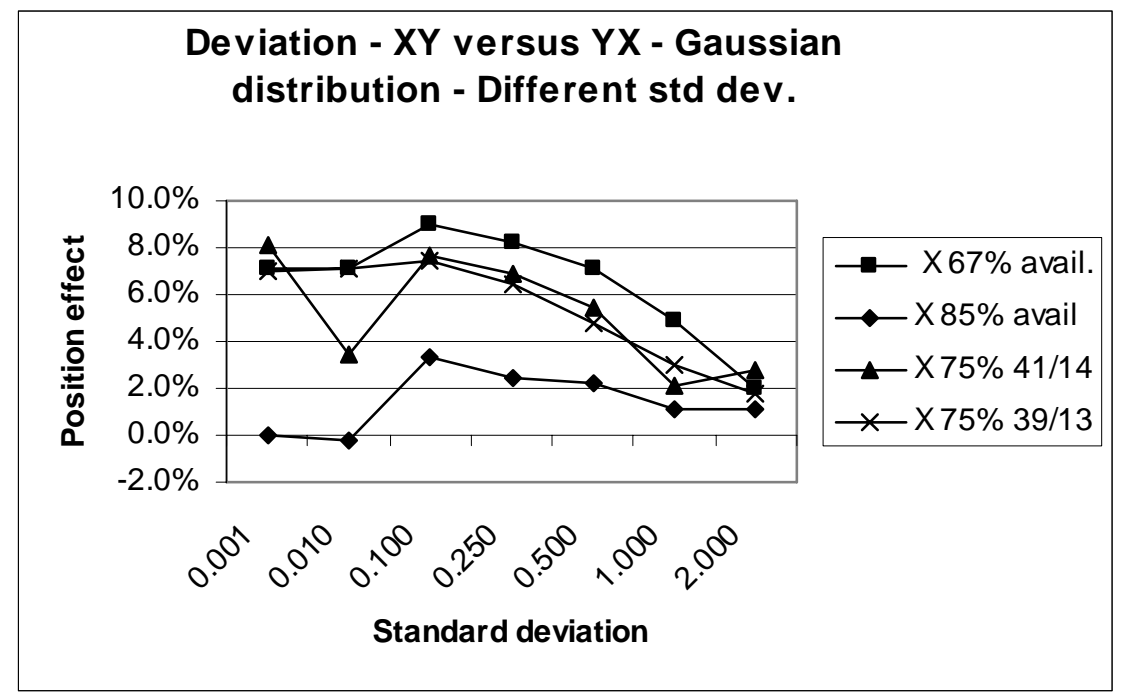

Figure 11: Results such as these reveal the difficulties inherent in the identification of reliable trends in the detailed behaviour of production simulation models.

\section{CONCLUSIONS}

1. The modelled productivity of a production line is highly dependent on the statistical distribution type assigned to each breakdown variable (eg Gaussian or triangular 
distributions of MTTF and MTTR). Statistical distributions with a broad range (such as Gaussian) will tend to result in low productivity. This is because occasional periods of low productivity will not necessarily be balanced by occasional periods of high productivity if the whole line is considered.

2. Although an increase in the range of MTTF and MTTR will generally result in a decrease in productivity, this trend can be severely disrupted at small values of standard deviation or if fixed mean values are used. If fixed or almost fixed values are used it is important that the values are selected very carefully to avoid setting up breakdown 'rhythms' which could result in unrealistically high or low productivity results. For the same reason fixed or small standard deviation values cannot necessarily be used to validate the performance of simulation models (This technique of variation reduction for model validation is in widespread use).

3. The modelling of any engineering system must consider whether the process involved at each step can be interrupted and later continued to produce a usable component. Many engineering processes such as casting, welding or painting cannot be interrupted and any mid operation breakdown will result in a scrap component.

4. The productivity of a production line involving unreliable but interchangeable machines is improved if the less reliable machines are positioned towards the end of the line. This effect diminishes as the reliability of the machines in question improves. The magnitude of this effect is strongly dependant on the statistical distribution type assigned to the breakdown parameters.

\section{REFERENCES}

[1] Ilar, T.; Powell, J.; Kaplan, A. (2007). The effect of process interruption and scrap on production simulation models, Proceedings of the Int. Conf. CARV, Toronto, Canada

[2] Selvaraj, N.; Rao, C. S. P. (2003). Simulation of machine breakdown in a pull production system operated by various control mechanisms, Proceedings of the Int. Conf. Modelling and Simulation, 485-490

[3] Bellgran, M.; Aresu, E. (2003). Handling disturbances in small volume production, Robotics and Computer Integrated Manufacturing, Vol. 19, 123-134

[4] Mittal, S.; Wang, H. P. (1992). Simulation of JIT Production to Determine Number of Kanbans, Int. Journal of Advanced Manufacturing Technology, Vol. 7, 292-305

[5] Johri, P. K.; Lipper, E. H.; Sengupta, B. (1985). Modelling and analysis of a production line with finite buffers and machine subject to breakdown, Systémes de production, Vol. 5, 471-483

[6] Shin, F.; Ram, B.; Gupta, A.; Yu, X.; Menassa, R. (2004). A decision tool for assembly line breakdown action, Proceedings of the Winter Simulation Conference, 1122-1127

[7] Ingemansson, A.; Bolmsjö, G. (2004). Improved efficiency with production disturbance reduction in manufacturing systems based on discrete-event simulation, Journal of Manufacturing Technology Management, Vol. 15, 267-279

[8] Ingemansson, A.; Ericsson, J.; Bolmsjö, G. (2003). Increased performance efficiency in manufacturing systems with production improvement techniques and discrete-event simulation, Proceedings of Int. Congress of Mechanical Engineering

[9] Law, A. M.; Kelton, W. D. (1991). Simulation, Modelling \& Analysis, McGraw-Hill, Boston

[10] Robinson, S. (2003). Simulation - The Practice of Model Development and Use, John Wiley \& Sons, Sussex 